\title{
An Interdisciplinary Framework for impacting older adults Health and Physical activity
}

\author{
Christopher J Dondzila ${ }^{1 *}$ and Elaine Van Doren ${ }^{2}$ \\ ${ }^{1}$ Department of Movement Science, Grand Valley State University, USA \\ ${ }^{2}$ Grand Valley State University, USA
}

*Corresponding author: Christopher J Dondzila, Department of Movement Science, Grand Valley State University, USA.

Received Date: August 15, 2019

Published Date: September 03, 2019

\begin{abstract}
The exploration of feasible and cost-effective strategies is warranted to mitigate rising healthcare costs and lessen the impact of chronic diseases, functional decline, and disability in older adults. The overwhelming sedentariness of older adults is accompanied by a lack of expertise by healthcare professionals in exercise programming that acknowledge factors influencing physical activity (PA) patterns. We present a framework for a nursing/exercise science interdisciplinary effort to increase PA and improve health in older adults via the delivery of individually tailored exercise programming for an 8-week intervention. Results from this study will be integral in translating effective interdisciplinary efforts across diverse healthcare settings.
\end{abstract}

Keywords: Older adults; Interdisciplinary; Health; Nursing; Physical activity

\section{Introduction}

Chronic diseases have an immense influence on well-being and healthcare expenditures in older adults, with the impact compounding in the presence of co-morbidities [1]. Accordingly, the exploration of new and cost-effective strategies is warranted to mitigate rising healthcare costs and lessen the impact of chronic diseases, functional decline, and disability, while promoting a pathway to longevity and high quality of life. Physical activity (PA) and exercise have long been shown to be strongly linked to a myriad of variables associated with aging [2], yet the majority of older adults are insufficiently active to obtain such benefits, even in the presence of general guidelines to accomplish so [3]. Thus, there is a practicality to intervening on PA as part of the healthy aging process, particularly in those who are at-risk or currently seeking treatment for chronic diseases.

Nurses typically have more interaction with patients than physicians do [4], representing massive potential to utilize this relationship as a conduit to quickly and efficiently deliver PA prescriptions to impact health in an at-risk population. However, there is a lack of expertise by healthcare professionals in exercise programming and factors influencing PA patterns [5]. Interdisciplinary efforts in healthcare teams provide a solution by congregating diverse professionals with unique expertise to work toward a common goal. More recently, there has been an urge to impress upon health professionals PA guidelines, benefits, and strategies to promote such behavior [6]. This is mirrored by the Exercise is Medicine $₫$ initiative to increase dialogue between patients and healthcare providers regarding PA as a treatment and refer to qualified exercise professionals when appropriate [7].

To explore the impactfulness of this interdisciplinary opportunity, a pilot study is being conducted to explore the efficacy of a nursing/exercise science interdisciplinary team to increase PA and improve health. Specifically, nursing students enrolled in a community nursing course had weekly rounds with older adults with chronic diseases residing in an independent-living, lowincome housing facility (serving as the healthcare practitioner, in this model) collaborated with exercise science professionals. The premise of this study is to utilize the routine interaction of the nursing students to deliver individually tailored exercise programming developed by an interdisciplinary team.

Within this 8-week intervention, nursing students conducted baseline health assessments and weekly follow-ups. The exercise science professional assessed baseline physical functioning (short 
physical performance battery), self-efficacy, and social support, and PA via a Fitbit(C) activity tracker. Those participants randomly assigned to the experimental group would receive weekly step goals (increasing $10 \%$ of the baseline step average each week), along with a resistance band and visual guide to performing upper and lower body exercises. Those randomized to the standard of care group would receive the general recommendation to increase PA levels to 10,000 steps/day. Baseline measures were re-assessed at the conclusion of the 8-week study.

It is hypothesized that this novel interdisciplinary intervention will increase PA and improve health in at-risk older adults. This framework has the healthcare practitioner merely passing along PA programming designed by the exercise science professional, which is facilitated by low-cost resources (activity trackers and resistance bands), thus overcoming barriers to PA (lack of knowledge and resources). The uniqueness is that there is no additional expertise/ training required by the healthcare professionals, and providing brief instruction takes very little time. This study will also elucidate barriers for this type of interdisciplinary effort and strategies to streamline the process moving forward. With favorable results, future studies should work on implementing this interprofessional model with a variety of professionals in a more formal healthcare setting.

\section{Acknowledgement}

None.

\section{Conflict of interest}

No Conflict of interest.

\section{References}

1. (2013) Centers for Disease Control and Prevention. The State of Aging and Health in America 2013. Atlanta, GA: Centers for Disease Control and Prevention, US Dept of Health and Human Services, USA.

2. Chodzko Zajko WJ, Proctor DN, Fiatarone Singh MA, Christopher T Minson, Claudio R Nigg, et al. (2009) Exercise and Physical Activity for Older Adults. Med Sci Sports Ex 41(7): 1510-1530.

3. Piercy KL, Troiano RP, Ballard RM (2018) The physical activity guidelines for Americans. JAMA 320(19): 2020-2028.

4. Khowaja Punjwani S, Smardo C, Hendricks MR, Lantos JD (2017) Physician-Nurse Interactions in Critical Care. Pediatrics 140(3): e20170670.

5. Cardinal BJ, Park EA, Kim M, Cardinal MK (2015) If Exercise is Medicine, where is Exercise in Medicine? Review of U.S. Medical Education Curricula for Physical Activity-Related Content. J Phys Act Health 12(9): 1336-1343.

6. King KM, Jaggers JR, Wintergerst K (2019) Strategies for Partnering with Health Care Settings to Increase Physical Activity Promotion. ACSM's Health \& Fitness Journal 23(4): 40-43.

7. Pojednic R, Bantham A, Arnstein F, Kennedy MA, Phillips E (2018) Bridging the gap between clinicians and fitness professionals: a challenge to implementing exercise as medicine. BMJ Open Sport Exerc Med 4(1): e000369. 